\title{
Crystalloid fluid therapy
}

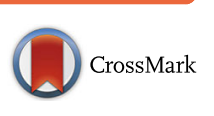

Sumeet Reddy ${ }^{1 *}$, Laurence Weinberg ${ }^{2,3}$ and Paul Young ${ }^{1,4}$

\begin{abstract}
This article is one of ten reviews selected from the Annual Update in Intensive Care and Emergency medicine 2016. Other selected articles can be found online at http://www.biomedcentral.com/collections/ annualupdate2016. Further information about the Annual Update in Intensive Care and Emergency Medicine is available from http://www.springer.com/ series/8901.
\end{abstract}

\section{Background}

"She filled his ancient veins with rich elixir. As he received it ... his wasted form renewed, appeared in all the vigor of bright youth, no longer lean and sallow, for new blood coursed in his well-filled veins" (Publius Ovidius Naso, Metamorphoses Book VII The Story of Medea and Jason, 8 AD).

Our desire to find solutions that rejuvenate and resuscitate is captured in the story of Medea revitalizing Jason's elderly father by filling his veins with a specially prepared elixir. Although no such elixir exists, intravenous fluids are an integral component of the multimodal resuscitation strategy used in medicine. Intravenous fluids were first administered over 180 years ago and despite their widespread use there remains uncertainty about their relative safety and efficacy.

Worldwide, there is variation in the prescribing of resuscitative intravenous fluids and the preferred choice of fluid appears to be based on local customs, marketing, fluid costs and availability [1]. The majority of intravenous fluids were introduced into clinical practice during an era where they did not undergo the same scrutiny as other drugs. Hence, there is a paucity of research in this area and only recently has there been an increase in academic interest in the comparative effectiveness of different intravenous fluids.

\footnotetext{
* Correspondence: sumeet.reddy@mrinz.ac.nz

${ }^{1}$ Medical Research Institute of New Zealand, 6021 Wellington, New Zealand Full list of author information is available at the end of the article
}

Here, we review the composition of different crystalloid fluids, potential pathophysiological responses following crystalloid fluid infusion, evidence from animal studies, observational studies, and interventional studies comparing crystalloid fluids, and suggest future directions for research on the comparative effectiveness of various crystalloid fluids.

\section{Unbuffered/unbalanced crystalloids}

The composition of $0.9 \%$ saline was first mentioned by Jakob Hamburger in the 1890s. It is unknown how $0.9 \%$ saline became known as 'normal saline'; however, use of term 'normal' may have contributed to the widespread acceptance of $0.9 \%$ saline into clinical practice. Despite being referred to as 'normal', $0.9 \%$ saline is not physiologically 'normal'. First, $0.9 \%$ saline has a higher chloride concentration than plasma. Second, $0.9 \%$ saline has a different strong ion difference (SID) to plasma. According to the Stewart physiochemical approach to describing acidbase balance, fluid $\mathrm{pH}$ is in part determined by the SID, which is the sum of the strong cation concentrations in the solution (e.g., sodium, potassium, magnesium), minus the sum of the strong anion concentrations in the solution (e.g., chloride and lactate). The SID of the extracellular fluid is approximately $40 \mathrm{mEq} / \mathrm{l}$, whereas the SID of $0.9 \%$ saline is zero. Following an infusion of $0.9 \%$ saline there is a net decrease in the plasma SID resulting in a metabolic acidosis.

$0.9 \%$ saline is often thought of as a relatively hypertonic solution because the sum of its osmotically active components gives a theoretical in vitro osmolality of $308 \mathrm{mosmol} / \mathrm{kg} \mathrm{H}_{2} \mathrm{O}(154 \mathrm{mmol} / \mathrm{l}$ sodium plus $154 \mathrm{mmol} /$ 1 chloride). However, $0.9 \%$ saline is more accurately referred to as an isotonic solution as its constituents - sodium and chloride - are only partially active, with an osmotic coefficient of 0.926 . The calculated in vivo osmolality (tonicity) of saline is $285 \mathrm{mosmol} / \mathrm{kgH}_{2} \mathrm{O}$, which is the same as plasma osmolality (tonicity).

\section{Buffered/balanced crystalloids}

Sydney Ringer's in vitro experiments in the 1880s on the influence of crystalloid fluid composition on cardiac 
contractility led to the recognition of a potential benefit of the addition of other inorganic constituents to sodium chloride solutions. Alexis Hartmann further modified Ringer's solution through the addition of sodium lactate to act as a buffering agent in an effort to combat acidosis in dehydrated pediatric patients.

Unlike $0.9 \%$ saline, the available buffered crystalloid solutions contain physiological or near physiological amounts of chloride. One of the key differences between $0.9 \%$ saline and buffered/balanced crystalloids is the presence of additional anions, such as lactate, acetate, malate and gluconate, which act as physiological buffers to generate bicarbonate. Further, buffered fluids, such as Hartmann's solution and Ringer's lactate, have near physiologically effective in vivo SIDs of 27 and $29 \mathrm{mEq} / \mathrm{l}$, respectively. In contrast, Plasma-Lyte $148^{\circ}$ has an effective SID in vivo of $50 \mathrm{mEq} / \mathrm{l}$. Despite the fact that buffered crystalloid fluids are designed to better mimic the composition of human plasma, no perfectly balanced or physiologically 'normal' crystalloid fluid is currently available (see Table 1).

Historically, sodium acetate was used during hemodialysis as an alternative to bicarbonate because of the incompatibility of bicarbonate with solutions containing calcium and magnesium salts. Early evidence suggested that sodium acetate solution was effective in restoring blood $\mathrm{pH}$ and plasma bicarbonate in patients suffering from metabolic acidosis [2]; however, a more recent study suggested that acetate was associated with hemodynamic instability, vasodilatation and negative inotropic affects in patients undergoing high volume renal replacement therapy (RRT) [3]. Concern about the potential for myocardial depression with acetate is supported by studies suggesting that acetate decreases myocardial contractilityand blood pressure in dogs [4] and impaired contractile function in anisolated perfused rat heart model [5]. Even the small quantity of acetate present in various dialysis fluids (usually $35 \mathrm{mmol} / \mathrm{l}$ ) can result in plasma acetate concentrations of 10 to 40 times the physiological level (50 to $100 \mu \mathrm{mol} / \mathrm{l}$ ) [6]. Use of acetated solutions as acircuit prime for cardiac patients undergoing cardiopulmonary bypass $(\mathrm{CPB})$ also results in short-livedsupra-physiological concentrations of acetate; however, it is not clear if these have any adverse clinical effects [7].

Despite these potential concerns, there are several theoretical advantages of using acetated solutions compared tolactate-containing crystalloids. Acetate is metabolized widely throughout the body, is not reliant entirely on hepaticmetabolism and is metabolized more rapidly than lactate [8]. A canine study showed that acetate metabolism was well-preserved in profound shock while lactate metabolism was significantly impaired [9]. Acetate metabolism does not result in changes in glucose or insulin

Table 1 Characteristics of common crystalloid solutions compared to human plasma

\begin{tabular}{|c|c|c|c|c|c|c|c|}
\hline & Plasma & 0.9 \% saline & $\begin{array}{l}\text { Compound sodium } \\
\text { lactate (lactate } \\
\text { buffered solution) }\end{array}$ & $\begin{array}{l}\text { Ringer's lactate } \\
\text { (lactate buffered } \\
\text { solution) }\end{array}$ & $\begin{array}{l}\text { lonosteril }{ }^{\oplus} \text { (acetate } \\
\text { buffered solution) }\end{array}$ & $\begin{array}{l}\text { Sterofundin ISO } \\
\text { (acetate \& malate } \\
\text { buffered solution) }\end{array}$ & $\begin{array}{l}\text { Plasma-Lyte } 148^{\oplus} \\
\text { (acetate \& gluconate } \\
\text { buffered solution) }\end{array}$ \\
\hline Sodium (mmol/l) & $136-145$ & 154 & 129 & 130 & 137 & 145 & 140 \\
\hline Potassium (mmol/l) & $3.5-5.0$ & & 5 & 4 & 4 & 4 & 5 \\
\hline Magnesium (mmol/l) & $0.8-1.0$ & & & & 1.25 & 1 & 1.5 \\
\hline Calcium (mmol/l) & $2.2-2.6$ & & 2.5 & 3 & 1.65 & 2.5 & \\
\hline Chloride (mmol/l) & $98-106$ & 154 & 109 & 109 & 110 & 127 & 98 \\
\hline Acetate (mmol/l) & & & & & 36.8 & 24 & 27 \\
\hline Gluconate $(\mathrm{mmol} / \mathrm{l})$ & & & & & & & 23 \\
\hline Lactate (mmol/l) & & & 29 & 28 & & & \\
\hline Malate (mmol/l) & & & & & & 5 & \\
\hline eSID (mEq/l) & 42 & & 27 & 28 & 36.8 & 25.5 & 50 \\
\hline $\begin{array}{l}\text { Theoretical osmolarity } \\
\text { (mosmol/l) }\end{array}$ & 291 & 308 & 278 & 273 & 291 & 309 & 295 \\
\hline $\begin{array}{l}\text { Actual or measured } \\
{ }^{a} \text { Osmolality } \\
\left(\text { mosmol/kg } \mathrm{H}_{2} \mathrm{O}\right)\end{array}$ & 287 & 286 & 256 & 256 & 270 & Not stated & 271 \\
\hline $\mathrm{pH}$ & $7.35-7.45$ & $4.5-7$ & $5-7$ & $5.0-7$ & $6.9-7.9$ & $5.1-5.9$ & $4-8$ \\
\hline
\end{tabular}

${ }^{a}$ Freezing point depression

Plasma-Lyte 148 manufactured by Baxter Healthcare, Toongabie, NSW, Australia

Ringer's Lactate manufactured by Baxter Healthcare, Deerfield, IL, USA

Hartmann's solution manufactured by Baxter Healthcare, Toongabie, NSW, Australia

Ionosteril manufactured by Fresenius Medical Care, Schweinfurt, Germany

Sterofundin ISO manufactured by B. Braun Melsungen AG, Melsungen, Germany 
concentrations, whereas exogenously administered lactate can be converted to glucose via gluconeogenesis resulting in hyperglycemia [10]. Acetate turnover shows no age-related differences [11], and acetate may protect against malnutrition by replacing fat as anoxidative fuel without affecting glucose oxidation, or causing hyperglycemia [10].

Little is known about the clinical effects of gluconate. Gluconate is largely excreted unchanged in the urine (80\%). In a recent Phase II evaluation of an acetate/ gluconate-based buffered solution (Plasma-Lyte $148^{\circ}$ ) versus abicarbonate buffered crystalloid fluid for $\mathrm{CPB}$ circuit priming, Plasma-Lyte $148^{\circ}$ was associated with an immediate increase in unmeasured anions of $>10 \mathrm{mEq} / \mathrm{l}$ (presumably acetate and/or gluconate), with residual elevations still present just prior to CPB cessation [12]. The clinical significance of elevated gluconate and/or acetate levels remains unclear in this setting.

Hartmann's solution or Ringer's lactate are hypotonic solutions with a calculated in vivo osmolality (tonicity) of approximately $254 \mathrm{mOsmol} / \mathrm{kgH}_{2} \mathrm{O}$. Perioperative administration of hypotonic fluids can represent a significant free water load that may not be easily excreted in the presence of the high anti-diuretic hormone concentrations commonly associated with physiological stress. Failure to excrete water in a timely fashion may result in postoperative positive fluid balance, edema, and weight gain. Hypotonic fluids are also contraindicated in patients with or at risk of cerebral edema.

In addition to differences in buffering agents, buffered solutions also vary in the presence and concentration of ancillary cations (sodium, potassium, calcium, magnesium), which means they are not biologically equivalent. Hartmann's solution contains $2 \mathrm{mmol} / \mathrm{l}$ of calcium and is contraindicated with blood or blood-related products due to concerns about precipitation and the possibility of coagulation and clot formation. A recent warning has been issued about mixing calcium-containing solutions, including Hartmann's solution or Ringer's lactate, with ceftriaxone causing the formation of the insoluble ceftriaxone calcium salt [13].

Although some authors have argued that hyperchloremia secondary to $0.9 \%$ saline is a benign and self-limiting phenomenon, there is some evidence that hyperchloremia is independently associated with adverse clinical outcomes [14]. The biological plausibility that $0.9 \%$ saline may affect renal function is supported by a cross-over study of 12 volunteers that reported significantly higher serum chloride levels, reduced renal artery blood velocity and reduced renal cortical tissue perfusion in subjects who received 21 of $0.9 \%$ saline over $1 \mathrm{~h}$ compared to those that had received Plasma-Lyte $148^{\circ}$ [15]. This study also found that although $0.9 \%$ saline and Plasma-Lyte $148^{\circ}$ expanded the intravascular volume to the same degree, $0.9 \%$ saline expanded the extracellular fluid volume significantly more than did Plasma-Lyte $148^{\circ}$ meaning that $0.9 \%$ saline may be more likely to result in fluid overload and interstitial edema.

Experimental research has identified possible hematological and gastrointestinal pathways that may be impaired with the use of $0.9 \%$ saline. Ex vivo testing of diluted whole blood reported that dilution with Ringer's lactate resulted in less impairment in thrombin generation and platelet activation when compared to $0.9 \%$ saline [16]. A swine study reported that metabolic acidosis significantly impaired gastropyloric motility by reducing pyloric contraction amplitude, which results in delayed gastric emptying or gastroparesis [17].

\section{Animal studies comparing $0.9 \%$ saline to buffered crystalloid fluids}

Recently, Zhou et al. reported decreased rates of acute kidney injury (AKI) and improved survival in rats receiving Plasma-Lyte ${ }^{\odot}$ compared to $0.9 \%$ saline in an animal model of sepsis [18]. In this experiment, 60 rats were randomized to receive $0.9 \%$ saline or Plasma-Lyte ${ }^{\circ}$ for $4 \mathrm{~h}(10 \mathrm{ml} / \mathrm{kg}$ for the first hour and $5 \mathrm{ml} / \mathrm{kg}$ over the next $3 \mathrm{~h}$ ) after $18 \mathrm{~h}$ of cecal ligation and puncture. Rats that received $0.9 \%$ saline had higher rates of AKI (100\% vs. $76 \%, p<0.05)$ and significantly worse AKI severity based on the Risk, Injury, Failure, Loss of kidney function, End-stage kidney disease (RIFLE) criteria creatinine definitions (RIFLE-I or F: $83 \%$ vs. $28 \%, p<0.001$ ). Histopathological and biomarkers (urine cystatin $\mathrm{C}$ and urine neutrophil gelatinase-associated lipocalin) of AKI were also significantly worse in rats that received $0.9 \%$ saline compared to rats that received Plasma-Lyte ${ }^{\circ}$.

The largest animal study comparing $0.9 \%$ saline versus buffered fluid was a swine model of hemorrhagic shock that randomized 116 pigs to crystalloid fluid replacement at different percentages of replacement $(0.9 \%$ saline at $14 \%$ replacement of blood loss; $0.9 \%$ saline at $100 \%$ replacement of blood loss; $0.9 \%$ saline at $300 \%$ replacement of blood loss; Ringer's lactate at $300 \%$ replacement of blood loss; Plasma-Lyte $\mathrm{A}^{\bullet}$ at $300 \%$ replacement of blood loss; Plasma-Lyte $\mathrm{R}^{\circ}$ at $300 \%$ replacement of blood loss) [19]. In a comparison of the different $300 \%$ treatment groups, a significant difference was found in survival at $24 \mathrm{~h}$ in pigs that received Ringer's lactate $(67 \%)$ compared to Plasma-Lyte $\mathrm{A}^{\circ}(30 \%)$. No difference was found in survival rates in $0.9 \%$ saline $(50 \%)$ or Plasma-Lyte $\mathrm{R}^{\bullet}(40 \%)$ groups.

\section{Observational studies comparing $0.9 \%$ saline to buffered crystalloid fluids}

The majority of evidence demonstrating potential adverse clinical effects with the use of $0.9 \%$ saline compared to buffered crystalloids originated from recent 
observational studies in critically unwell and surgical patients [20-22]. In a single center, open-label, sequential 6-month study of 1533 critically ill patients, the change from standard chloride-liberal fluids $(0.9 \%$ saline, $4 \%$ succinylate gelatin or $4 \%$ albumin) to chloride-restrictive fluids (Hartmann's solution, Plasma-Lyte $148^{\circ}$ and $20 \%$ albumin) was associated with a significant decrease in the risk of developing RIFLE-defined AKI (odds ratio [OR] $0.52,95 \%$ CI $0.37-0.75, p<0.01$ ) and requirements for RRT while in the intensive care unit (ICU) (OR 0.52, $95 \%$ CI, 0.33-0.81, $p=0.004$ ) [20]. No difference between groups was found in hospital mortality and hospital or ICU length of stay. A recently published extended analysis over the 12 months before and after the strategy change, which included 2994 patients, reported persistently lower rates of AKI according to the Kidney Disease: Improving Global Outcomes (KDIGO) creatinine definitions and decreased requirements of RRT [23]. Because multiple changes in fluids occurred simultaneously in this observational study it is not possible to determine what component of the fluid change strategy (if any) was responsible for the observed changes.

The two largest studies that have assessed the effects of buffered versus unbuffered crystalloid fluid were retrospective observational studies with patient data collected from centralized health-economic databases [21, 22]. The most recent study was conducted in non-surgical, adult patients with the International Classification of Disease, Ninth Edition Clinical Modification (ICD-9$\mathrm{CM}$ ) codes for sepsis who were receiving vasopressors in the ICU by day two, and had received three consecutive days of antibiotics and had had a blood culture [21]. In total 53,448 patients were identified from 360 hospitals over five years. Of this cohort, only 3365 patients $(6.4 \%)$ had received some "balanced fluids" during their first two hospital days. This sample was compared with a propensity-matched group of 3365 patients who had "not received balanced fluids" (received either $0.9 \%$ saline or $5 \%$ dextrose). Patients who had received balanced fluids had a significantly lower in-hospital mortality (19.6\% vs. $22.8 \%$; relative risk [RR] 0.86, $95 \%$ CI $0.78-0.94, p=0.001$ ) compared to the group that had not received balanced fluids. No difference was found between groups in acute renal failure (defined by ICD-9-CM codes), need for dialysis and hospital or ICU length of stay. The vast majority of patients in the balanced group had received a mixture of intravenous fluids and it was reported that less than $1 \%$ of patients in the balanced fluid group had exclusively received balanced fluids. On further analysis, patients were stratified by the proportion of balanced to unbalanced fluid they had received. The relative risk of in-hospital mortality was progressively lower among patients who received a greater proportion of balanced fluid.
A similar retrospective study was conducted in adult patients who had undergone non-traumatic, open, general surgical abdominal operations who had exclusively received either $0.9 \%$ saline or a balanced fluid (defined as a calcium-free buffered fluid: Plasma-Lyte $148^{\circ}$ or Plasma-Lyte $A^{\circ}$ ) on the day of surgery [22]. In total 271,189 patients from approximately 600 hospitals had received fluids on the day of surgery. Of these patients, 30,994 received $0.9 \%$ saline and 926 received balanced fluid. Propensity matching was used to mitigate for baseline group imbalances, which included a significantly higher proportion of minorities, less commercial insurance, greater proportion of patients from non-teaching hospitals, greater proportion of admissions via the emergency department and significantly higher rates of $\mathrm{co}^{-}$ morbidities (based on ICD-9-CM codes), such as renal failure, diabetes and congestive heart failure in patients that had exclusively received $0.9 \%$ saline. On matched analysis, patients who exclusively received balanced fluid had a decreased risk of major complications (OR 0.79, $95 \%$ CI 0.66-0.97, $p<0.05)$ including need for blood transfusion (1.8\% vs. $11.5 \%, p<0.001)$ and need for dialysis $(1.0 \%$ vs. $4.8 \%, p<0.001)$. However, those in the balanced fluid group had higher rates of minor gastrointestinal complications (OR 1.45; 95 \% CI 1.17$1.79, p<0.05)$ and longer hospital lengths of stay (6.4 vs. 5.9 days, $p<0.001$ ).

Overall, existing data from observational studies suggest that the use of high chloride, unbuffered crystalloid fluid may be associated with major complications following surgery and increased mortality in critically ill patients with sepsis. However, due to the retrospective nature of these studies and potential for unmeasured confounding, it is not possible to establish whether using buffered crystalloid fluid instead of $0.9 \%$ saline is beneficial or harmful on the basis of observational studies.

\section{Interventional studies comparing $0.9 \%$ saline to buffered crystalloid fluids}

Until 2015, all interventional studies comparing $0.9 \%$ saline to buffered crystalloid had a small sample size $(n<100)$ and focused primarily on short term physiological or biochemical outcomes (see Table 2). A systematic review and meta-analysis published in 2014 identified 28 prospective, randomized controlled trials with at least 20 adult participants that had compared the effects of different crystalloid fluids [24]. Twentythree studies that explored acid-base balance reported that $0.9 \%$ saline was associated with decreased serum $\mathrm{pH}$, elevated serum chloride levels and decreased bicarbonate levels. In 11 studies that explored renal function, based on urine volume or serum creatinine, no significant difference was found between fluids. In 
Table 2 Summary of the key interventional clinical studies that have compare $0.9 \%$ saline to buffered crystalloid fluid in adult patients

\begin{tabular}{|c|c|}
\hline & Design, setting and participants \\
\hline \multicolumn{2}{|c|}{ Acutely unwell population } \\
\hline $\begin{array}{l}\text { The SPLIT trial. } \\
2015 \text { [27] }\end{array}$ & $\begin{array}{l}\text { Multicentre, double-blind, cluster randomised, double crosso } \\
\text { comparing } 0.9 \% \text { saline with Plasma-Lyte } 148^{\circ} ; n=2262\end{array}$ \\
\hline $\begin{array}{l}\text { Smith et al. } \\
2015 \text { [28] }\end{array}$ & $\begin{array}{l}\text { Single centre, double-blind RCT comparing } 0.9 \% \text { saline with } \\
\text { Plasma-Lyte } A^{\oplus} \text { in critically ill trauma patients; } n=18\end{array}$ \\
\hline
\end{tabular}

Young et al. $\quad$ Single centre, double-blind RCT comparing $0.9 \%$ saline with 2014 [29] Plasma-Lyte $A^{\oplus}$ in patient presenting to ED with severe acute trauma; $n=46$

Cieza et al. 2013 [30]

Hasman et al. Single centre, double-blind RCT comparing either $0.9 \%$ saline, 2012 [31] Ringer's lactate or Plasma-Lyte ${ }^{\circledast}$ in patients presenting to ED with dehydration; $n=90$

Van Zyl et al. Multicentre, double-blind RCT of Ringer's lactate versus $0.9 \%$ 2012 [32] saline in patients presenting to ED with diabetic ketoacidosis; $n=54$

Mahler et al. Single centre, double-blind RCT comparing either $0.9 \%$ saline with 2011 [33] Plasma-Lyte $A^{\oplus}$ in patients presenting to ED with diabetic ketoacidosis; $n=45$

Wu et al. Multicentre, open label RCT comparing $0.9 \%$ saline with Ringer's 2011 [34] lactate in patients diagnosed with acute pancreatitis; $n=40$

Cho et al. Multicentre, single-blind RCT of Ringer's lactate versus $0.9 \%$ 2007 [35] saline in patients presenting to ED with rhabdomyolysis; $n=28$

Key Findings

- There was no significant difference between group

in rates of $\mathrm{AKI}$ or $\mathrm{AKI}$ requiring RRT

- There was no significant difference between groups in survival to day 90

- Patients receiving $0.9 \%$ saline had significantly lower serum chloride and bicarbonate concentration - Patients receiving Plasma-Lyte $A^{\oplus}$ had a quicker fibrin build up and cross linking (a angle) at 6 hours after infusion - No difference between groups in coagulation tests or blood products received at 6 hours

- Patients receiving $0.9 \%$ saline had an increase in serum chloride concentration and decrease in serum $\mathrm{pH}$ - No significant differences in mortality hospital length of stay, blood transfusion requirements or utilization of resources

- Patients receiving $0.9 \%$ saline had lower serum pH at 2 and 4 hours

- No difference between in serum creatinine, lactate or potassium concentration

- Patients receiving $0.9 \%$ saline had a significantly lower serum $\mathrm{pH}$ and lower serum bicarbonate concentration

- No difference between groups in chloride, potassium, or sodium concentrations

- There was no significant difference between groups in time interval for correction of acidosis - Patient receiving $0.9 \%$ saline a significantly shorter time to lower blood glucose

- No difference between groups in hospital length of stay

- Patients receiving $0.9 \%$ saline had significantly higher serum chloride and lower bicarbonate concentration

- Patients receiving Ringer's lactate had lower rates of SIRS and lower CRP concentration at 24 hours - No difference between groups in development of complications or hospital length of stay

- Patients receiving $0.9 \%$ saline had a significantly higher serum chloride and sodium concentration and lower serum $\mathrm{pH}$

- There was no significant difference between groups in time interval for normalisation of creatine kinase

Surgical population

The SPLITMajor Surgery trial. 2015
Prospective phase 4 , single centre blinded study investigating the safety and efficacy of using $0.9 \%$ saline with Plasma-Lyte ${ }^{\circledast} 148$ as fluid therapy in adult patients undergoing major surgery; $n=1100$
Potura et al 2015 [36]
Single centre, open label RCT comparing $0.9 \%$ saline with Elomel Isoton ${ }^{\oplus}$ (low chloride, acetate buffered crystalloid) in patients undergoing renal transplantation; $n=150$
- There was no significant difference between groups in rates of AKI

- There were no significant difference between groups in the development of postoperative complications or length of hospital stay - Patients who received $0.9 \%$ saline developed a transient hyperchloremic metabolic acidosis on postoperative day 1

- Significantly more patients receiving $0.9 \%$ saline required intra-operative inotrope support - Patients receiving $0.9 \%$ saline had a significantly lower base excess and higher serum chloride concentration

- No difference between groups in post-operative urine output, creatinine, blood urea nitrogen or need for RRT 
Table 2 Summary of the key interventional clinical studies that have compare $0.9 \%$ saline to buffered crystalloid fluid in adult patients (Continued)

\begin{tabular}{ll}
\hline Song et al. & Single centre, open label RCT comparing $0.9 \%$ saline with \\
2015 [37] & Plasma-Lyte ${ }^{\circledast}$ in patients undergoing spinal surgery; $n=50$
\end{tabular}

Hafizah et al. Single centre, open label RCT comparing $0.9 \%$ saline with 2015 [38] Sterofundin ${ }^{\oplus}$ ISO patients undergoing neurosurgery (low chloride, acetate buffered crystalloid); $n=30$

Kim et al. 2013 [39]

Modi et al. $2012[40]$

Heidari et al. 2011 [41]

Hadimioglu et al. 2008 [42]

Khajavi et al. 2008 [43]

Chin et al. 2006 [44]

Karaca et al. 2006 [45]

Chanimov et al. 2006 [46]

O'Malley et al. 2005 [47]

Takil et al. 2002 [48]

Waters et al. 2001 [49]

lactate and 5\% saline in patients undergoing lower abdomina surgery; $n=90$

Single centre, single-blinded RCT comparing $0.9 \%$ saline with

transurethral prostatectomy under spinal anesthesia; $n=60$

Single centre, double-blinded RCT comparing $0.9 \%$ saline with Ringer's lactate in patients undergoing Cesarean section; $n=40$ lactate in patients undergoing renal transplantation; $n=51$

Single centre, open label RCT comparing $0.9 \%$ saline with Ringer's lactate in patients undergoing spinal surgery; $n=30$
Single centre, blinded RCT comparing either $0.9 \%$ saline with Plasma-Lyte ${ }^{\circledast}$ in patients undergoing renal transplantation; $n=60$

Single centre, double-blind RCT comparing $0.9 \%$ saline with Ringer's lactate in patients undergoing renal transplantation; $n=74$
- Patients receiving $0.9 \%$ saline had lower $\mathrm{pH}$, base excess, and bicarbonate concentration and higher serum chloride concentration

- Patients receiving Plasma-Lyte ${ }^{\circledast}$ had significantly higher urine output

- No difference between groups in rotation thromboelastometry analysis, estimated blood loss or transfusion requirements

- Patients receiving $0.9 \%$ saline had a significantly lower serum $\mathrm{pH}$ and higher serum chloride and sodium concentration

- Patients receiving $0.9 \%$ saline had lower pH and base excess values

- No difference between groups in post-operative urine output, creatinine or need for RRT

- Patients receiving $0.9 \%$ saline had lower serum pH and base excess values

- No difference between groups in post-operative urine output or creatinine

- A higher proportion of patients that had received $0.9 \%$ saline had experienced vomiting 6 hours post-operatively

- Patients receiving $0.9 \%$ saline had an increase in serum chloride concentration and decrease in serum $\mathrm{pH}$ - Patients receiving Ringer's lactate had a significantly increased serum lactate concentration - There was no significant difference between in postoperative creatinine or need for RRT

- Patient receiving $0.9 \%$ saline had a significantly lower serum $\mathrm{pH}$ and higher serum potassium concentration at the end of the operation

- No difference between groups in serum urea sodium or potassium concentration

- Dextrose $5 \%$ resulted in significant, albeit transient hyperglycemia, even in non-diabetic patients

- No difference between groups nausea, vomiting, Ringer's lactate and $4 \%$ gelatin polysuccinate in patients undergoing dizziness and post spinal hearing loss.

- No difference between groups in inotrope requirements

- No significant differences in the Apgar scores at 1 and 5 min or infant well-being

Single centre, double blind RCT comparing $0.9 \%$ saline with Ringer's • Significantly more patients receiving $0.9 \%$ saline required intra-operative treatment for metabolic acidosis and hyperkalemia

- No difference between groups in post-operative urine output, creatinine or need for RRT

- Patients receiving $0.9 \%$ saline had an increase in serum chloride, sodium concentration and decrease in serum $\mathrm{pH}$ - No difference between groups in intraoperative hemodynamic variables or hospital and ICU lengths of stay $\begin{array}{ll}\text { Single centre, double-blind RCT comparing } 0.9 \% \text { saline with Ringer's } & \text { - Patients receiving } 0.9 \% \text { saline had an increase in } \\ \text { lactate in patients undergoing abdominal aortic aneurysm surgery; } & \text { serum chloride, sodium concentration and }\end{array}$ $n=66$ decrease in serum $\mathrm{pH}$

- Patients receiving $0.9 \%$ saline received a greater volume of platelets

- No difference between groups in estimated blood loss, postoperative complications, hospital and ICU lengths of stay 
Table 2 Summary of the key interventional clinical studies that have compare $0.9 \%$ saline to buffered crystalloid fluid in adult patients (Continued)

\begin{tabular}{|c|c|c|}
\hline $\begin{array}{l}\text { Scheingraber } \\
\text { et al. } 1999[50]\end{array}$ & $\begin{array}{l}\text { Single centre, open label RCT comparing } 0.9 \% \text { saline with Ringer's } \\
\text { lactate in patients undergoing gynecologic surgery; } n=24\end{array}$ & $\begin{array}{l}\text { - Patients receiving } 0.9 \% \text { saline had an increase in } \\
\text { serum chloride concentration and decrease in } \\
\text { serum pH }\end{array}$ \\
\hline $\begin{array}{l}\text { Ramanathan } \\
\text { et al. } 1984 \text { [551] }\end{array}$ & $\begin{array}{l}\text { Single centre, open label RCT comparing } 0.9 \% \text { saline with Ringer's } \\
\text { lactate, Ringer's lactate with dextrose } 5 \% \text { and Plasma-Lyte } A^{\oplus} \\
\text { in patients undergoing Cesarean section; } n=60\end{array}$ & $\begin{array}{l}\text { - Patients receiving } 0.9 \% \text { saline had an decrease in } \\
\text { serum } \mathrm{pH} \\
\text { - No difference between groups in blood pressure or } \\
\text { inotrope requirements }\end{array}$ \\
\hline
\end{tabular}

$A K I$ acute kidney injury, CRP C-reactive protein, ED emergency department, $R C T$ randomized control trial, $R R T$ renal replacement therapy, SIRS systemic inflammatory response syndrome, The SPLIT trial $0.9 \%$ Saline versus Plasma-Lyte $148^{\oplus}$ for Intensive Care Unit fluid Therapy trial, The SPLIT- Major Surgery trial $0.9 \%$ Saline or Plasma-Lyte ${ }^{\circledR} 148$ as fluid therapy in adult patients undergoing major surgery trial

three surgical studies $(n=156)$ that reported volumes of red blood cells transfused, patients who had received Ringer's lactate required significantly less volume of red blood cells than those who had received $0.9 \%$ saline (RR 0.42, $95 \%$ CI $0.11-0.73$ ). No differences were found between Ringer's lactate and $0.9 \%$ saline in requirements for transfusion or operative blood loss, except in an exploratory sub-group analysis of "high-risk" patients that showed increased blood loss with the use of $0.9 \%$ saline in patients that were at increased risk of bleeding.

Based on the published evidence prior to 2014, the National Institute for Health and Care Excellence (NICE) guidelines on intravenous fluid therapy in adults in hospital currently recommend the use of crystalloids that contain sodium in the range $130-154 \mathrm{mmol} / \mathrm{l}$ for fluid resuscitation [25]. However, the guidelines state that the available evidence at the time of writing was limited and of "poor" quality. Research todate has been in heterogeneous populations and has been underpowered to allow for differences to be detected inclinically significant outcome measures. The NICE committee specifically identified that research comparing balanced solutions to $0.9 \%$ saline for fluid resuscitation was a high priority.

\section{The SPLIT program}

The $0.9 \%$ saline vs. Plasma-Lyte $148^{\circ}$ for intravenous fluid therapy research program is an investigator-initiated, bi-national collaborative research program investigating the comparative effectiveness of $0.9 \%$ saline versus a buffered crystalloid as intravenous fluid therapy. Specific details on the study design, methods of analyzing and reporting of the research program have been published previously [26]. At the time of writing, four of the six planned studies in critically unwell and elective surgical patients have been completed.

The largest study was the $0.9 \%$ Saline versus PlasmaLyte $148^{\circ}$ for Intensive care fluid Therapy (SPLIT) trial. The SPLIT trial was a multicenter, blinded, cluster randomized, double crossover study that compared PlasmaLyte $148^{\circ}$ with $0.9 \%$ saline as the routine ICU intravenous fluid [27]. All ICU patients needing crystalloid fluid therapy were eligible to be included. Patients who were on dialysis, expected to require RRT within six hours and patients admitted to the ICU solely for organ donation or for palliative care were excluded. In total, 2262 patients in four New Zealand tertiary ICUs over a 28-week period were enrolled and analyzed, with 1152 patients assigned to receive Plasma-Lyte $148^{\circ}$ and 1110 assigned to receive $0.9 \%$ saline. The two groups of patients had similar admission diagnoses and baseline characteristics. There were no differences between patients who received Plasma-Lyte $148^{\circ}$ compared to $0.9 \%$ saline in rates of AKI (9.6\% vs. $9.2 \%$ [difference $0.4 \%, 95 \%$ CI $-2.1 \%-2.9 \%$; RR 1.04, $95 \%$ CI $0.80-1.36, p=0.77])$ or requirements for RRT (3.3 \% vs. $3.4 \%$ [difference $0.1 \%$, $95 \%$ CI $-1.5 \%-1.4 \%$; RR 0.96, $95 \%$ CI 0.62-1.50, $p=0.91])$. There were also no significant differences in need for mechanical ventilation, readmission to the ICU, ICU length of stay or in hospital mortality.

Two single-centered, pilot, nested cohort studies were also conducted in patients enrolled in the SPLIT trial. The first of these studies evaluated 251 adults who had undergone cardiac surgery: 131 were allocated to PlasmaLyte $148^{\circ}$ and 120 were allocated to $0.9 \%$ saline. No difference was found between groups in postoperative chest drain output or in the proportion of patients developing a major postoperative complication (death, myocardial infarction, new focal neurological deficit or renal failure requiring dialysis). However, fewer patients in the $0.9 \%$ saline group required blood products (packed red blood cells, fresh frozen plasma, platelets or cryoprecipitate) compared to patients in the Plasma-Lyte $148^{\circ}$ group (18.3\% vs. $30.5 \%, p=0.03)$. The second nested cohort study compared gastrointestinal feeding intolerance in 69 patients (35 assigned to receive Plasma-Lyte $148^{\circ}$ and 34 to receive $0.9 \%$ saline) expected to require mechanical ventilation for greater than $48 \mathrm{~h}$ and receiving enteral nutrition exclusively by a nasogastric tube. Despite no difference between groups in the proportion of patients with gastrointestinal feeding intolerance (defined as high gastric residual volume, diarrhea or vomiting while receiving nasogastric feeding in the ICU), a significantly lower proportion of patients in the Plasma-Lyte $148^{\circ}$ 
group developed high gastric residual volumes (11.4 \% vs. $32.4 \%, p=0.04$ ).

Finally, a prospective single center blinded study investigated the safety and efficacy of using $0.9 \%$ saline or Plasma-Lyte 148 as fluid therapy in adult patients undergoing major surgery. Trial fluid was used intraoperatively and postoperatively for three consecutive days. Inclusion criterion included patients undergoing surgery of at least two hours duration and requiring at least one overnight stay. Patients with end-stage renal disease and those undergoing liver or renal transplantation were excluded. The primary outcome measure was the proportion of patients with either acute kidney injury or failure based on creatinine levels in accordance with RIFLEcriteria during the index hospital admission. Intraoperatively, there was $100 \%$ compliance with the trial protocol. A total of 746 patients received Plasma-Lyte $148^{\circ}$ and 634 patients received $0.9 \%$ saline. Patients in both groups had similar baseline characteristics with respect to age, sex, body mass index, American Society of Anesthesiologists (ASA) status, types and number of comorbidities, duration and types of surgery. The median amount of trial fluid received was greater in the PlasmaLyte $148^{\circ}$ group: $2000 \mathrm{ml}$ (interquartile range [IQR] 1000, $2000)$ vs. $1925 \mathrm{ml}(1000,2000)$ in the $0.9 \%$ saline group $(p=0.007)$. Patients receiving $0.9 \%$ saline developed a transient hyperchloremic metabolic acidosis on postoperative Day 1 compared to patients receiving Plasma-Lyte $148^{\circ}$. Postoperatively, there were no differences in the incidence of AKI between the groups: $52(10.9 \%)$ patients in the Plasma-Lyte $148^{\circ}$ group developed postoperative AKI compared to $59(9.3 \%)$ patients in the $0.9 \%$ saline group ( $p=0.41,95 \%$ CI 0.6-1.2). Patients who developed AKI were older, had larger volumes of fluid both intraoperatively and on postoperative Day 1, and had greater fluid balances intraoperatively and on postoperative Day 1 . There were no differences in the development of postoperative complications between the groups. Median lengths of stay were similar between treatment groups: Plasma-Lyte $148^{\circ}$ 5.0 days (2.77-8.98) vs. $0.9 \%$ saline 5.0 days (2.81-9.04).

\section{Conclusion}

Intravenous fluid therapy is a ubiquitous intervention in critically ill patients. While pre-clinical and observational data raise the possibility that the choice of crystalloid fluid therapy may affect patient-centered outcomes, there are currently no convincing data from interventional studies demonstrating that this is the case. Recent data suggest that $0.9 \%$ saline and Plasma-Lyte 148 result in similar rates of renal complications when used for fluid therapy in patients undergoing major surgery and in ICU patients. Further large randomized controlled trials are needed to assess the comparative effectiveness of $0.9 \%$ saline and balanced/buffered crystalloids in high-risk populations and to measure clinical outcomes such as mortality. Moreover, given the widespread use of a range of balanced/buffered crystalloids in current clinical practice, high quality studies comparing the various buffered crystalloids available are also needed.

\section{Competing interests}

Laurence Weinberg has received research funding from Baxter Healthcare. Paul Young report receiving honoraria (<USD\$5000) from Baxter Healthcare Pty Ltd for consulting activities. No other disclosures are reported.

\section{Authors' contributions}

SR, LW, and PY, have contributed in the literature review and drafting of the manuscript. All authors read and approved the final manuscript.

\section{Declarations}

Publication of this article was funded by the Medical Research Institute of New Zealand.

\section{Author details}

${ }^{1}$ Medical Research Institute of New Zealand, 6021 Wellington, New Zealand.

${ }^{2}$ Austin Hospital, Department of Anesthesia, Melbourne, VIC, Australia.

${ }^{3}$ Departments of Surgery and Anesthesia, University of Melbourne,

Perioperative Pain Medicine Unit, Melbourne, VIC, Australia. ${ }^{4}$ Wellington

Regional Hospital, Intensive Care Unit, Wellington, New Zealand.

Published online: 15 March 2016

\section{References}

1. Finfer S, Liu B, Taylor C, Bellomo R, et al. Resuscitation fluid use in critically ill adults: an international cross-sectional study in 391 intensive care units. Crit Care. 2010;14:R185.

2. Eliahou HE, Feng PH, Weinberg U, laina A, Reisin E. Acetate and bicarbonate in the correction of uraemic acidosis. BMJ. 1970;4:399-401.

3. Schrander-vd Meer AM, Ter Wee PM, Kan G, et al. Improved cardiovascular variables during acetate free biofiltration. Clin Nephrol. 1999;51:304-9.

4. Kirkendol RL, Pearson JE, Bower JD, Holbert RD. Myocardial depressant effects of sodium acetate. Cardiovasc Res. 1978;12:127-36.

5. Jacob AD, Elkins N, Reiss OK, Chan L, Shapiro Jl. Effects of acetate on energy metabolism and function in the isolated perfused rat heart. Kidney Int. 1997;52:755-60

6. Coll E, Perez-Garcia R, Rodriguez-Benitez P, et al. Clinical and analytical changes in hemodialysis without acetate. Nefrologia. 2007;27:742-8.

7. Davies PG, Venkatesh B, Morgan TJ, et al. Plasma acetate, gluconate and interleukin-6 profiles during and after cardiopulmonary bypass: a comparison of Plasma-Lyte 148 with a bicarbonate-balanced solution. Crit Care. 2011;15:R21.

8. Mudge $\mathrm{GH}$, Manning JA, Gilman A. Sodium acetate as a source of fixed base. Proc Soc Exp Biol Med. 1949;71:136-8.

9. Kveim M, Nesbakken R. Utilization of exogenous acetate during canine haemorrhagic shock. Scand J Clin Lab Invest. 1979;39:653-8.

10. Akanji AO, Bruce MA, Frayn KN. Effect of acetate infusion on energy expenditure and substrate oxidation rates in non-diabetic and diabetic subjects. Eur J Clin Nutr. 1989:43:107-15.

11. Skutches $C L$, Holroyde CP, Myers RN, Paul P, Reichard GA. Plasma acetate turnover and oxidation. J Clin Invest. 1979;64:708-13.

12. Morgan TJ, Power G, Venkatesh B, Jones MA. Acid-base effects of a bicarbonate-balanced priming fluid during cardiopulmonary bypass: comparison with Plasma-Lyte 148. A randomised single-blinded study. Anaesth Intensive Care. 2008;36:822-9.

13. Murney P. To mix or not to mix - compatibilities of parenteral drug solutions. Aust Prescr. 2008;31:98-101.

14. Neyra JA, Canepa-Escaro F, Li X, et al. Association of Hyperchloremia With Hospital Mortality in Critically III Septic Patients. Crit Care Med. 2015;43:1938-44.

15. Chowdhury AH, Cox EF, Francis ST, Lobo DN. A randomized, controlled, double-blind crossover study on the effects of $2-L$ infusions of $0.9 \%$ saline and plasma-lyte(R) 148 on renal blood flow velocity and renal cortical tissue perfusion in healthy volunteers. Ann Surg. 2012;256:18-24.

16. Brummel-Ziedins K, Whelihan MF, Ziedins EG, Mann KG. The resuscitative fluid you choose may potentiate bleeding. J Trauma. 2006;61:1350-8. 
17. Tournadre JP, Allaouchiche B, Malbert CH, Chassard D. Metabolic acidosis and respiratory acidosis impair gastro-pyloric motility in anesthetized pigs. Anesth Analg. 2000;90:74-9.

18. Zhou F, Peng ZY, Bishop JV, Cove ME, Singbartl K, Kellum JA. Effects of fluid resuscitation with $0.9 \%$ saline versus a balanced electrolyte solution on acute kidney injury in a rat model of sepsis. Crit Care Med. 2014:42:270-8.

19. Traverso LW, Lee WP, Langford MJ. Fluid resuscitation after an otherwise fatal hemorrhage: I. Crystalloid solutions. J Trauma. 1986;26:168-75.

20. Yunos NM, Bellomo R, Hegarty C, Story D, Ho L, Bailey M. Association between a chloride-liberal vs chloride-restrictive intravenous fluid administration strategy and kidney injury in critically ill adults. JAMA. 2012;308:1566-72

21. Raghunathan K, Shaw A, Nathanson B, et al. Association between the choice of IV crystalloid and in-hospital mortality among critically ill adults with sepsis. Crit Care Med. 2014:42:1585-91.

22. Shaw AD, Bagshaw SM, Goldstein SL, et al. Major complications, mortality, and resource utilization after open abdominal surgery: $0.9 \%$ saline compared to Plasma-Lyte. Ann Surg. 2012;255:821-9.

23. Yunos NM, Bellomo R, Glassford N, Sutcliffe H, Lam Q, Bailey M. Chloride liberal vs. chloride-restrictive intravenous fluid administration and acute kidney injury: an extended analysis. Intensive Care Med. 2015;41:257-64.

24. Orbegozo Cortes D, Rayo Bonor A, Vincent JL. Isotonic crystalloid solutions: a structured review of the literature. Br J Anaesth. 2014;112:968-81.

25. Padhi S, Bullock I, Li L, Stroud M. Intravenous fluid therapy for adults in hospital: summary of NICE guidance. BMJ. 2013;347:f7073.

26. Reddy SK, Young PJ, Beasley RW, et al. Overview of the study protocols and statistical analysis plan for the Saline versus Plasma-Lyte 148 for Intravenous Fluid Therapy (SPLIT) research program. Crit Care Resusc. 2015;17:29-36.

27. Young $P$, Bailey $M$, Beasley $R$, et al. Effect of a buffered crystalloid solution vs saline on acute kidney injury among patients in the intensive care unit. The SPLIT randomized clinical trial. JAMA. 2015;314:1701-10.

28. Smith CA, Gosselin RC, Utter GH, et al. Does saline resuscitation affect mechanisms of coagulopathy in critically ill trauma patients? An exploratory analysis. Blood Coagul Fibrinolysis. 2015;26:250-4.

29. Young JB, Utter GH, Schermer CR, et al. Saline versus Plasma-Lyte A in initial resuscitation of trauma patients: a randomized trial. Ann Surg. 2014;259:255-62.

30. Cieza JA, Hinostroza J, Huapaya JA, Leon CP. Sodium chloride $0.9 \%$ versus Lactated Ringer in the management of severely dehydrated patients with choleriform diarrhoea. J Infect Dev Ctries. 2013;7:528-32.

31. Hasman H, Cinar O, Uzun A, Cevik E, Jay L, Comert B. A randomized clinical trial comparing the effect of rapidly infused crystalloids on acid-base status in dehydrated patients in the emergency department. Int J Med Sci. 2012:9:59-64

32. Van Zyl DG, Rheeder P, Delport E. Fluid management in diabetic-acidosis Ringer's lactate versus normal saline: a randomized controlled trial. QJM. 2012;105:337-43.

33. Mahler SA, Conrad SA, Wang H, Arnold TC. Resuscitation with balanced electrolyte solution prevents hyperchloremic metabolic acidosis in patients with diabetic ketoacidosis. Am J Emerg Med. 2011;29:670-4.

34. Wu BU, Hwang JQ, Gardner TH, et al. Lactated Ringer's solution reduces systemic inflammation compared with saline in patients with acute pancreatitis. Clin Gastroenterol Hepatol. 2011;9:710-7.

35. Cho YS, Lim H, Kim SH. Comparison of lactated Ringer's solution and $0.9 \%$ saline in the treatment of rhabdomyolysis induced by doxylamine intoxication. Emerg Med J. 2007;24:276-80.

36. Potura $E$, Lindner $G$, Biesenbach $P$, et al. An acetate-buffered balanced crystalloid versus $0.9 \%$ saline in patients with end-stage renal disease undergoing cadaveric renal transplantation: a prospective randomized controlled trial. Anesth Analg. 2015;120:123-9.

37. Song JW, Shim JK, Kim NY, Jang J, Kwak YL. The effect of $0.9 \%$ saline versus plasmalyte on coagulation in patients undergoing lumbar spinal surgery; a randomized controlled trial. Int J Surg. 2015;20:128-34.

38. Hafizah M, Liu CY, Ooi JS. Normal saline versus balanced-salt solution as intravenous fluid therapy during neurosurgery: Effects on acid-base balance and electrolytes. J Neurosurg Sci 2016. (in press)

39. Kim SY, Huh KH, Lee JR, Kim SH, Jeong SH, Choi YS. Comparison of the effects of normal saline versus Plasmalyte on acid-base balance during living donor kidney transplantation using the Stewart and base excess methods. Transplant Proc. 2013;45:2191-6.

40. Modi MP, Vora KS, Parikh GP, Shah VR. A comparative study of impact of infusion of Ringer's Lactate solution versus normal saline on acid-base balance and serum electrolytes during live related renal transplantation. Saudi J Kidney Dis Transpl. 2012;23:135-7.

41. Heidari SM, Saryazdi H, Shafa A, Arefpour R. Comparison of the effect of preoperative administration of Ringer's solution, normal saline and hypertonic saline $5 \%$ on postoperative nausea and vomiting: a randomized, double blinded clinical study. Pak J Med. 2011:27:771-4.

42. Hadimioglu N, Saadawy I, Saglam T, Ertug Z, Dinckan A. The effect of different crystalloid solutions on acid-base balance and early kidney function after kidney transplantation. Anesth Analg. 2008;107:264-9.

43. Khajavi MR, Etezadi F, Moharari RS, et al. Effects of normal saline vs. lactated ringer's during renal transplantation. Ren Fail. 2008;30:535-9.

44. Chin KJ, Macachor J, Ong KC, Ong BC. A comparison of $5 \%$ dextrose in 0. $9 \%$ normal saline versus non-dextrose-containing crystalloids as the initia intravenous replacement fluid in elective surgery. Anaesth Intensive Care. 2006:34:613-7.

45. Karaca BSM, Yildiz TS, Ozkarakas H, Toker K. Effects of various loading solutions on postspinal hearing loss. J Turk Anesthesiol Reanim Soc. 2006:36:156-61.

46. Chanimov M, Gershfeld S, Cohen ML, Sherman D, Bahar M. Fluid preload before spinal anaesthesia in Caesarean section: the effect on neonatal acidbase status. Eur J Anaesthesiol. 2006;23:676-9.

47. O'Malley CM, Frumento RJ, Hardy MA, et al. A randomized, double-blind comparison of lactated Ringer's solution and $0.9 \% \mathrm{NaCl}$ during renal transplantation. Anesth Analg. 2005;100:1518-24.

48. Takil A, Eti Z, Irmak P, Yilmaz Gogus F. Early postoperative respiratory acidosis after large intravascular volume infusion of lactated ringer's solution during major spine surgery. Anesth Analg. 2002;95:294-8.

49. Waters JH, Gottlieb A, Schoenwald P, Popovich MJ, Sprung J, Nelson DR. Normal saline versus lactated Ringer's solution for intraoperative fluid management in patients undergoing abdominal aortic aneurysm repair: an outcome study. Anesth Analg. 2001;93:817-22.

50. Scheingraber S, Rehm M, Sehmisch C, Finsterer U. Rapid saline infusion produces hyperchloremic acidosis in patients undergoing gynecologic surgery. Anesthesiology. 1999;90:1265-70.

51. Ramanathan S, Masih AK, Ashok U, Arismendy J, Turndorf H. Concentrations of lactate and pyruvate in maternal and neonatal blood with different intravenous fluids used for prehydration before epidural anesthesia. Anesth Analg. 1984;63:69-74. 